\title{
BMJ Open What is the best catheter lock solution in preventing catheter-related blood infections? A protocol for a Bayesian network meta-analysis of randomised controlled trials
}

\author{
Fangping Dang, ${ }^{1}$ Huiju Li, ${ }^{1}$ Jinhui Tian, ${ }^{2}$ Ruijuan Wang, ${ }^{1}$ Jingjie Ren ${ }^{1}$
}

To cite: Dang F, Li H, Tian J, et al. What is the best catheter lock solution in preventing catheter-related blood infections? A protocol for a Bayesian network meta-analysis of randomised controlled trials. BMJ Open 2019;9:e030019. doi:10.1136/ bmjopen-2019-030019

- Prepublication history and additional material for this paper are available online. To view, please visit the journal (http:// dx.doi.org/10.1136/bmjopen2019-030019).

Received 27 February 2019 Revised 7 May 2019 Accepted 31 May 2019
Check for updates

(c) Author(s) (or their employer(s)) 2019. Re-use permitted under CC BY-NC. No commercial re-use. See rights and permissions. Published by BMJ.

${ }^{1}$ School of Nursing, Lanzhou University, Lanzhou, China ${ }^{2}$ Evidence-based Medicine Center, Lanzhou University, Lanzhou, China

Correspondence to Huiju Li; lihj@lzu.edu.cn

\section{ABSTRACT}

Introduction Catheter-related bloodstream infection (CRBSI) is a major complication after central venous catheter insertion, which is associated with significant morbidity, mortality and additional medical costs. Many lock solutions for CRBSI have been evaluated. However, using traditional pairwise meta-analyses to summarise the evidence does not allow the inclusion of data from treatments that have not been compared head to head, which could impact the precision of pooled estimates in a meta-analysis. Therefore, we evaluated the efficacy and safety of the different lock solutions for CRBSI through a network meta-analysis.

Methods and analysis The primary outcome of this network meta-analysis is the CRBSI. The secondary outcomes are exit-site infection and catheter-related thrombosis. We will search the PubMed, Embase, Web of Science and the Cochrane Library databases for recent relevant meta-analysis and their reference lists to include randomised controlled trials (RCTs) that compared lock solutions for CRBSI prevention. Two individuals will independently extract data from each included RCT according to a predesigned Excel spreadsheet and will assess the methodological quality using the Cochrane risk of bias tool. We will analyse the data using WinBUGS (V.1.4.3) and Stata (V.15.0). We will also estimate the pooled direct and indirect effects for all lock solutions using the network meta-analysis.

Ethics and dissemination As the present meta-analysis is performed based on previous published studies, no ethical approval and patient safety considerations are required. This study commenced on 18 January 2019 , and its expected completion date is 1 December 2019. We will disseminate the results of our network meta-analysis through an international peer-reviewed journal.

PROSPERO registration number CRD42019121089.

\section{INTRODUCTION}

The central venous catheter (CVC) plays an important role in the care of patients with critical illness, on haemodialysis with cancer or who require parenteral nutrition. However, catheter-related bloodstream infection
Strengths and limitations of this study

- This review is anticipated to be the first network meta-analysis to address the important concern of which lock solution is most effective for catheter-related bloodstream infection.

- This network meta-analysis has a comprehensive literature search strategy involving restriction of studies to randomised controlled trials, duplicate assessment of eligibility and risk of bias and data abstraction.

- We will use the Grade of Recommendations Assessment, Development and Evaluation to assess the quality of treatment effect estimates from the network meta-analysis.

- Only included studies written in English may lead to publication bias.

(CRBSI), which is a main complication of CVCs, may increase the morbidity, mortality and medical costs, and prolong hospitalisations. ${ }^{1}{ }^{2}$ CRBSIs are the most expensive healthcare-associated infections, with an average cost of US $\$ 45814$ per episode. ${ }^{3}$ Therefore, the prevention of CRBSI is one of the major challenges in the routine care of CVC patients.

Consequently, several measures have been developed to prevent CRBSI, including antimicrobial dressings, catheter impregnation, antiseptic agents and education and training of medical workers. ${ }^{4-6}$ Recently, the use of catheter lock solutions has been associated with improved clinical outcomes and reduction of CRBSI after CVC insertion. ${ }^{78}$

The catheter lock solution is the injection of antimicrobials, wherein antibiofilms are instilled in the lumen of the CVC to eradicate organisms and prevent biofilm formation, thereby eliminating the source of CRBSI. ${ }^{9-11}$ At present, the most common lock solution 
is the use of heparin and saline to flush the lumen of the catheter. $^{12}$

There are several systematic reviews that focused on different catheter lock solutions for CRBSI in CVC patients. ${ }^{13-16}$ A previous meta-analysis ${ }^{13}$ included 6 randomised controlled trials (RCTs) and 431 patients and showed that taurolidine reduced the incidence of CRBSI without obvious adverse effects and bacterial; however, it did not conclude whether it is associated with a higher risk for catheter-related thrombosis compared with heparin. The studies by Zhao et al and Rahhal et al $l^{14}$ reported that the ethanol lock is effective in reducing the incidence of CRBSI in haemodialysis and paediatric patients compared with heparin. The study by Zacharioudakis $e a^{16}{ }^{16}$ study included 23 RCTs and 2896 patients who were predominantly adult patients undergoing haemodialysis (16/23 studies), and they suggested that antimicrobial lock solutions led to a $69 \%$ reduction in the CRBSI rate and $32 \%$ reduction in the rate of exit-site infections compared with heparin.

However, the comparative effectiveness of all available lock solutions has never been examined. Traditional systematic reviews cannot compare multiple treatments simultaneously. A network meta-analysis (NMA) can compare all available treatments in one synthesis even if the treatments have not been investigated directly in a RCT.

Hence, this protocol describes the methods for an NMA to assess which lock solution (or their combination) is most likely to be effective for patients with CVC. We will also estimate the treatment effects through direct and indirect comparisons, and generate a ranking according to the efficacy and safety outcomes of all solutions.

\section{METHODS AND ANALYSIS}

This protocol follows the Preferred Reporting Items for Systematic Reviews and Meta-Analysis Protocols ${ }^{17}$ and Preferred Reporting Items for Systematic Reviews and Meta-Analyses extension for NMA checklist for reporting systematic review protocols and NMA. ${ }^{18} 19$ This review was registered in the International Prospective Register of Systematic Reviews (PROSPERO: CRD42019121089).

\section{Eligibility criteria}

The eligibility criteria are prespecified according to the $\mathrm{P}$ refer to the patient or population. The I refer to the intervention/exposure. The $\mathrm{C}$ refer to the comparison/ control. The $\mathrm{O}$ refer to the outcome. The $\mathrm{S}$ refer to the study design. (PICO $(\mathrm{S}))$ criteria.

\section{Types of participants}

All patients who underwent CVC insertion will be included. We will not impose an age restriction. Studies will be included if they use antimicrobial lock solutions for CRBSI. The participants must be using a tunnelled or non-tunnelled CVC as venous access for $\geq 48$ hours.
Patients with artery catheter or pacing wires were excluded regardless of the cause and duration of catheter use.

\section{Types of interventions}

RCTs of antimicrobial lock solutions used to prevent CRBSI will be included, regardless of whether the antimicrobials were tested between themselves (head-to-head) or against placebo/control intervention such as heparin. For antimicrobials, antibiotics, citrate, taurolidine and alcohol will be included regardless of their concentration. The anticoagulants (eg, heparin, citrate or EDTA (minocycline-EDTA lock solution)) could be given in all antimicrobial lock solutions. The comparator can be placebo, heparin or a different catheter lock solution. Comparing interventions of the same lock solution with different concentrations is beyond the scope of this study. For example, if the treatment group was administered $1000 \mathrm{IU} / \mathrm{mL}$ heparin, whereas the control group was administered $5000 \mathrm{IU} / \mathrm{mL}$ heparin, this study will be excluded.

We included all RCTs that met the above-mentioned inclusion criteria and included a control group (indirect evidence) or at least two interventions (direct evidence).

\section{Types of outcome}

Included studies must report CRBSI as one of the end points (defined below).

\section{Primary outcome}

CRBSI is defined according to the Infectious Diseases Society of America recommendations. ${ }^{20}$ A definite CRBSI is diagnosed in a patient with bacteraemia who has an intravascular device and $>1$ positive blood culture with a recognised pathogen or common commensal, with confirmation of infection by isolation of the same organism following culture of the catheter tip, or a differential time to positivity for centrally and peripherally drawn blood cultures of $\geq 2$ hours.

\section{Secondary outcomes}

Catheter-related thrombosis

According to the Doppler ultrasound, CVC-related thrombosis is diagnosed in case of non-compressibility of the vein, visualisation of an echogenic intraluminal mass and absence of respiratory variation or monophasic flow within 30 days after insertion of the CVC and within 24 hours after removal of the CVC. ${ }^{21}$ In our NMA, the inability to use the catheter at a blood flow of $>200 \mathrm{~mL} /$ min despite additional flushing and intraluminal thrombolysis was used for the definition of thrombosis. ${ }^{22}$

\section{Exit-site infection}

According to the Centers for Disease Control (Atlanta, Georgia, USA), the exit-site infection is the appearance of erythema, tenderness and/or induration within $2 \mathrm{~cm}$ of the dialysis catheter exit site with or without purulent exudates; microbiological exit-site infection is characterised by presence of exudates leading to microorganism's growth in the culture. ${ }^{23}$ 
The study authors will use a variety of definitions for bleeding and CRBSI. If several definitions of CRBSI are presented in the same study, our preferred definition will be according to the Infectious Diseases Society of America recommendations. ${ }^{20}$ The secondary outcomes will be defined according to the author definition of each study.

\section{Types of studies}

Eligible studies are RCTs assessing the effect of lock solutions in the prevention of CRBSI.

\section{Search strategy}

We will search PubMed, Embase, the Cochrane Library and Web of Science from inception until January 2019. We will create the search strategy to combine relevant Medical Subject Headings and keywords with synonyms and names of generic and brand names of all catheter lock solutions. The search strategy will be developed by $\mathrm{JT}$, who has $>10$ years of experience as an information specialist. We have provided a draft of a full electronic search strategy for PubMed in online supplementary file named 'Search Strategy'. We will also track the references in the included articles and relevant systematic reviews/meta-analyses to identify additional relevant studies. Meanwhile, we will perform weekly research in the above-mentioned electronic databases to identify any newly published articles until the submission of the present study. Non-English studies and duplicate publications of original research will be excluded. We will also exclude ongoing RCTs without extractable data.

\section{Study selection}

Literature search records will be imported into EndNoteX8 literature management software (Thomson Reuters, New York, New York, USA). Two researchers (FD and JT) will independently review the title and abstract of the studies and exclude those that clearly did not meet the inclusion criteria. Then, the remaining studies will be identified by reviewing the full text according to the prespecified inclusion criteria. Any disagreements will be resolved by discussion or through a third reviewer (HL).

\section{Data extraction}

Data will be independently extracted by two authors (FD and JT) from each included RCT according to a predesigned Excel spreadsheet created in Microsoft Excel 2016 (Microsoft, Redmond, Washington, USA). The study data will include study and publication details, participants and intervention characteristics as well as outcome. If disagreement persists, a third reviewer (HL) will make the decision.

\section{Risk of bias assessment}

The methodological risk of bias of included RCTs will be assessed according to the Cochrane Collaboration Risk of Bias Tool $^{24}$ including the method of random sequence generation (selection bias), allocation concealment, blinding of participants and personnel (performance bias), blinding of outcome assessment (detection bias), incomplete outcome data (attrition bias), selective reporting (reporting bias) and other biases. These items will be evaluated as having high, low or unclear risk of bias. Any disagreements will be resolved through a discussion and reaching a consensus with the third reviewer (HL).

\section{Data synthesis and statistical methods}

We planned this NMA to assess which lock solution for CRBSI is most effective. We will construct the networks for the primary and secondary outcomes separately.

\section{Bayesian NMA}

Bayesian NMA will be performed in WinBUGS 1.4.3 (V.1.4, Medical Research Council, UK, and Imperial College of Science, Technology and Medicine, University of Cambridge, UK) by using the Markov chain Monte Carlo method, and the other analyses will be performed and presented through Stata V.15.0 using the mvmeta command. The following two different chains will be used:

- chain 1: treatment effect: $(\mathrm{d}(\mathrm{k})=0) ; \mathrm{SD}=1 ; \mathrm{mu}(\mathrm{i})=0$;

- chain 2: treatment effect: $(\mathrm{d}(\mathrm{k})=-1) ; \quad \mathrm{SD}=4$; $\mathrm{mu}(\mathrm{i})=-3$, where $\mathrm{d}(\mathrm{k})=$ treatmenteffect of experimental intervention ' $k$ ' compared with reference and $m u(i)=$ treatment effect of the experimental intervention compared with control in the trial 'i'. Model fit will be determined based on the deviance information criteria (DIC) for each outcome measure. It is best to choose the lowest model of DIC (differences $>3$ are considered meaningful). ${ }^{25}$ Two Markov chains will run simultaneously with different arbitrarily chosen initial values. We will generate the simulations for each chain if the convergence will be found to be adequate. Then, these simulations will be discarded as 'burn-in'. To ensure convergence, the model convergence will be assessed by trace plots. ${ }^{26}$

\section{Methods for indirect and direct comparisons}

We will conduct the separate pairwise meta-analyses using the random-effects model to compare all the interventions with available direct evidence. Dichotomous data will be determined by using OR with $95 \%$ CI. Continuous outcomes will be analysed using weighted mean differences or standardised mean differences if different measurement scales are used. To present indirect comparisons of the catheter lock solutions, a network diagram will be drawn. Trials will be excluded if they are not connected by any treatments. The common comparator will be heparin as it is the current standard of care. ${ }^{27}$ In this network plot, nodes represent different interventions and edges represent the head-to-head comparisons between interventions. The size of the node reflects the sample size of the intervention, and the thickness of the edge reflects the number of included trials.

\section{Dealing with missing data}

We will attempt to obtain missing data from authors of the included RCTs by mail. We will explore the potential 
impact of the missing data by performing a sensitivity analyses and record the number of patients 'loss to follow-up'. We will also extract data from studies that report outcomes using the intention-to-treat analysis.

\section{Treatment ranking}

To estimate the percentage of efficacy of each catheter lock solution for being at each possible rank, the surface under the cumulative ranking curve (SUCRA) values, which are expressed as percentages will be used. The SUCRA value is $100 \%$ for the best treatment and $0 \%$ for the worst treatment. The higher the SUCRA score, the more efficacious the treatment is expected to be. ${ }^{28} 29$

\section{Assessment of heterogeneity}

Clinical and methodological heterogeneity will be assessed by carefully examining the study population characteristics, interventions and outcomes of the included trials and comparing fit of the fixed-effects model and random-effects model. The statistical heterogeneity within each pairwise comparison will be measured by using the $\mathrm{I}^{2}$ index. ${ }^{30}$ Substantial heterogeneity will be considered where $\mathrm{I}^{2}$ is $>50 \%$. If considerable heterogeneity is found, network meta-regression or subgroup analysis will be used to explore possible sources of heterogeneity.

\section{Subgroup and sensitivity analyses}

Subgroup analyses and network meta-regression were planned to perform according to the type of catheter, catheter insertion site, catheter days/lock time, age and study quality. We will perform sensitivity analyses of the outcomes to determine the sensitivity of results to changes in the original assumptions by excluding trials with a high risk of bias in seven items, and excluding trials where the criterion of CRBSI diagnosis does not meet the above-mentioned definition.

\section{Assessment of inconsistency}

Inconsistency means the presence of disagreement between the different sources of evidence. ${ }^{31}$ We will evaluate the inconsistency between direct and indirect evidence when a loop is formed between treatments. The node-split method will be used to explore where the inconsistency is found. ${ }^{32}$ We will attempt to identify inconsistencies jointly from all possible sources in the network using the design-by-treatment interaction model and $\mathrm{I}^{2}$ index, when possible. ${ }^{33} 34$

\section{Publication bias}

When each direct treatment comparison and outcome contains a sufficient number of studies (at least 10 studies), we will assess the possibility of publication bias by visually examining the asymmetry of funnel plots and Egger's regression test. ${ }^{24}$ We will use a comparison-adjusted funnel plot to explore the potential small-study effects in the network and use contour-enhanced funnel plots to examine whether the funnel plot asymmetry is caused by a publication bias. ${ }^{35}$ If the publication bias is found, we will fit a selection model to obtain relative effects 'adjusted' for the impact of publication bias. ${ }^{36}$

\section{Quality of the evidence}

We will propose the following Grade of Recommendations Assessment, Development and Evaluation (GRADE) four-step approach ${ }^{37}$ to assess the quality of treatment effect estimates from NMA: (1) present direct and indirect treatment estimates for each comparison of the evidence network; (2) rate the quality of each direct and indirect effect estimate; (3) present the NMA estimate for each comparison of the evidence network and (4) rate the quality of each NMA effect estimate. According to the GRADE group, we will classify the quality of evidence into the following four levels: high quality, moderate quality, low quality and very low quality.

\section{Potential limitations of the planned work}

Some potential limitations of this NMA are predictable. First, excluding non-English studies may cause a publication bias. Second, we will only include RCT publications to support our intention to include only high-quality evidence, which indicate that there is a possibility that the study population will not be representative of the overall population. Besides, we only included patients with CRBSI defined according to the Infectious Diseases Society of America recommendations, which will increase the risk of bias and heterogeneity. Finally, if the number of included studies is small, our ability to explore heterogeneity, conduct a meta regression analysis and even perform NMA is limited. Meanwhile, if the degree of clinical heterogeneity is substantial, the internal validity will be threatened.

\section{Ethics and dissemination}

Given that the present meta-analysis is performed based on previous published studies, no ethical approval and patient safety considerations are required. This study commenced on 18 January 2019, and its expected completion date is 1 December 2019. We will disseminate the results of our network meta-analysis through an international peer-reviewed journal.

Contributors FD and JT conceived and designed this network meta-analysis. FD, RW and JR were involved in the acquisition and analysis of the data. FD and $\mathrm{HL}$ interpreted the results. FD and JT drafted this protocol. All authors have read and provided feedback and consented to the content of the article as submitted.

Funding The authors have not declared a specific grant for this research from any funding agency in the public, commercial or not-for-profit sectors.

Competing interests None declared.

Patient consent for publication Not required.

Provenance and peer review Not commissioned; externally peer reviewed.

Open access This is an open access article distributed in accordance with the Creative Commons Attribution Non Commercial (CC BY-NC 4.0) license, which permits others to distribute, remix, adapt, build upon this work non-commercially, and license their derivative works on different terms, provided the original work is properly cited, appropriate credit is given, any changes made indicated, and the use is non-commercial. See: http://creativecommons.org/licenses/by-nc/4.0/. 


\section{REFERENCES}

1. Olaechea PM, Palomar M, Álvarez-Lerma F, et al. Morbidity and mortality associated with primary and catheter-related bloodstream infections in critically ill patients. Rev Esp Quimioter 2013;26:21-9.

2. Dibb MJ, Abraham A, Chadwick PR, et al. Central venous catheter salvage in home parenteral nutrition catheter-related bloodstream infections: long-term safety and efficacy data. JPEN J Parenter Enteral Nutr 2016;40:699-704.

3. Zimlichman E, Henderson D, Tamir O, et al. Health care-associated infections: a meta-analysis of costs and financial impact on the US health care system. JAMA Intern Med 2013;173:2039-46.

4. Musu M, Finco G, Mura P, et al. Controlling catheter-related bloodstream infections through a multi-centre educational programme for intensive care units. J Hosp Infect 2017;97:275-81.

5. Chong HY, Lai NM, Apisarnthanarak A, et al. Comparative efficacy of antimicrobial central venous catheters in reducing catheter-related bloodstream infections in adults: abridged cochrane systematic review and network meta-analysis. Clin Infect Dis 2017;64(suppl_2):S 131-S140.

6. Edwards M, Rickard CM, Rapchuk I, et al. A pilot trial of bordered polyurethane dressings, tissue adhesive and sutureless devices compared with standard polyurethane dressings for securing shortterm arterial catheters. Crit Care Resusc 2014;16:175.

7. Zacharioudakis IM, Zervou FN, Arvanitis M, et al. Antimicrobial lock solutions as a method to prevent central line-associated bloodstream infections: a meta-analysis of randomized controlled trials. Clinical Infectious Diseases 2014;59:1741-9.

8. Norris LB, Kablaoui F, Brilhart MK, et al. Systematic review of antimicrobial lock therapy for prevention of central-line-associated bloodstream infections in adult and pediatric cancer patients. Int $J$ Antimicrob Agents 2017;50:308-17.

9. Chaftari AM, Hachem R, Szvalb A, et al. A Novel Non-Antibiotic Nitroglycerin Based Catheter Lock Solution for the Prevention of Intraluminal Central Venous Catheter Infections in Cancer Patients. Antimicrobial agents and chemotherapy 2017. AAC-00091.

10. Khalil GM, Azqul MM. Risk factors and microbial profile of central venous catheter related blood stream infection in medical cardiac care units, National Heart Institute, Egypt. Egypt Heart J 2018;70:361-4.

11. Labriola L, Pochet JM. Any use for alternative lock solutions in the prevention of catheter-related blood stream infections? The journal of vascular access 2017;18(1_suppl):S34-S38.

12. López-Briz E, Ruiz Garcia V, Cabello JB, et al. Heparin versus $0.9 \%$ sodium chloride locking for prevention of occlusion in central venous catheters in adults. Cochrane Database Syst Rev 2018;31.

13. Liu $Y$, Zhang AQ, Cao L, et al. Taurolidine lock solutions for the prevention of catheter-related bloodstream infections: a systematic review and meta-analysis of randomized controlled trials. PLoS One 2013;8:e79417.

14. Zhao T, Liu H, Han J. Ethanol lock is effective on reducing the incidence of tunneled catheter-related bloodstream infections in hemodialysis patients: a systematic review and meta-analysis. Int Urol Nephrol 2018;50:1643-52.

15. Rahhal R, Abu-El-Haija MA, Fei L, et al. Systematic review and metaanalysis of the utilization of ethanol locks in pediatric patients with intestinal failure. JPEN J Parenter Enteral Nutr 2018;42:690-701.

16. Zacharioudakis IM, Zervou FN, Arvanitis M, et al. Antimicrobial lock solutions as a method to prevent central line-associated bloodstream infections: a meta-analysis of randomized controlled trials. Clin Infect Dis 2014:59:1741-9.

17. Moher D, Shamseer L, Clarke M, et al. Preferred reporting items for systematic review and meta-analysis protocols (PRISMA-P) 2015 statement. Syst Rev 2015;4:1.
18. Shamseer L, Moher D, Clarke M, et al. Preferred reporting items for systematic review and meta-analysis protocols (PRISMA-P) 2015: elaboration and explanation. BMJ 2015;349:97647.

19. Hutton B, Salanti G, Caldwell DM, et al. The PRISMA extension statement for reporting of systematic reviews incorporating network meta-analyses of health care interventions: checklist and explanations. Ann Intern Med 2015;162:777-84.

20. O'Grady NP, Alexander M, Burns LA, et al. Guidelines for the prevention of intravascular catheter-related infections. Clin Infect Dis 2011;52:e162-e193.

21. Baxter GM, Kincaid W, Jeffrey RF, et al. Comparison of colour Doppler ultrasound with venography in the diagnosis of axillary and subclavian vein thrombosis. Br J Radiol 1991;64:777-81.

22. Sofroniadou S, Revela I, Kouloubinis A, et al. Ethanol combined with heparin as a locking solution for the prevention of catheter related blood stream infections in hemodialysis patients: A prospective randomized study. Hemodial Int 2017;21:498-506.

23. Mermel LA. Infectious Diseases Society of America, American College of Critical Care Medicine, Society for Healthcare Epidemiology of America. Guidelines for the management of intravascular catheter-related infections. J Intraven Nurs 2001;24:180-205.

24. Higgins JPT, Sterne JAC, Savović J, et al. A revised tool for assessing risk of bias in randomized trials. Cochrane Database Syst Rev 2016;10(Suppl 1):29-31.

25. Spiegelhalter DJ, Best NG, Carlin BP, et al. Bayesian measures of model complexity and fit. Journal of the Royal Statistical Society: Series B 2002;64:583-639.

26. Brooks SP, Gelman A. General methods for monitoring convergence of iterative simulations. Journal of computational and graphical statistics 1998;7:434-55.

27. Correa Barcellos F, Pereira Nunes B, Jorge Valle L, et al. Comparative effectiveness of $30 \%$ trisodium citrate and heparin lock solution in preventing infection and dysfunction of hemodialysis catheters: a randomized controlled trial (CITRIM trial). Infection 2017; $45: 139-45$

28. Chaimani A, Higgins JP, Mavridis D, et al. Graphical tools for network meta-analysis in STATA. PLoS One 2013;8:e76654.

29. Salanti G, Ades AE, loannidis JP. Graphical methods and numerical summaries for presenting results from multiple-treatment metaanalysis: an overview and tutorial. J Clin Epidemiol 2011;64:163-71.

30. Higgins JP, Thompson SG, Deeks JJ, et al. Measuring inconsistency in meta-analyses. BMJ 2003;327:557-60.

31. Salanti G, Del Giovane C, Chaimani A, et al. Evaluating the quality of evidence from a network meta-analysis. PLoS One 2014;9:e99682.

32. Dias S, Welton NJ, Caldwell DM, et al. Checking consistency in mixed treatment comparison meta-analysis. Stat Med 2010;29(78):932-44.

33. Higgins JP, Jackson D, Barrett JK, et al. Consistency and inconsistency in network meta-analysis: concepts and models for multi-arm studies. Res Synth Methods 2012;3:98-110.

34. Jackson D, Barrett JK, Rice S, et al. A design-by-treatment interaction model for network meta-analysis with random inconsistency effects. Stat Med 2014;33:3639-54.

35. Peters JL, Sutton AJ, Jones DR, et al. Contour-enhanced metaanalysis funnel plots help distinguish publication bias from other causes of asymmetry. J Clin Epidemiol 2008;61:991-6.

36. Mavridis D, Welton NJ, Sutton A, et al. A selection model for accounting for publication bias in a full network meta-analysis. Stat Med 2014;33:5399-412.

37. Puhan MA, Schünemann $\mathrm{HJ}$, Murad MH, et al. A GRADE Working Group approach for rating the quality of treatment effect estimates from network meta-analysis. BMJ 2014;349:g5630. 